\title{
Study of serum Ceruloplasmin, Malondialdehyde (MDA) and hs-CRP as biomarkers of severity in Psoriasis
}

\author{
1. K Prashanth, Assistant Professor, Department of Biochemistry, Rajiv Gandhi Institute of \\ Medical Sciences [RIMS], Adilabad, Telangana State, India.
}

Submission : 25-11-2020

Review : 30-11-2020

Acceptance 2-12-2020

\section{MohdInayatulla Khan, Assistant Professor, Department of Physiology, Rajiv Gandhi Institute of Medical Sciences [RIMS], Adilabad, Telangana State, India. \\ Corresponding Author: *Dr.MohdInayatulla Khan, Flat No 4, Building No I, RIMS quarters, RIMS Medical college, Dasnapur, Adilabad-504001. Telangana State. Email: drkhan123@rediffmail.com Mobile: 09948959937}

\begin{abstract}
Background : Psoriasis is one of the common diseases diagnosed in dermatology clinics. It is characterized by erythematous and sharply demarcated papules covered by silvery micaceous scales. Although, etiology is an unclear genetic predisposition seen in $50 \%$ of cases. This study aimed to determine the role of malondialdehyde (MDA), ceruloplasmin, and C-Reactive Protein (hs-CRP) as markers of severity of the disease.
\end{abstract}

Methods: $\mathrm{N}=75$ patients were selected and divided into three categories of different severity based on PASI scores. Those with PASI score of $<10$ were mild cases included in group I and with PASI Scores of 11-15 were moderate cases included in group II and PASI scores $>15$ were in group III. $\mathrm{N}=25$ age and sex-matched taken from healthy people were included in group IV controls.

Results: correlation coefficient $(r)$ values were calculated between PASI and biomarkers. Of the biomarkers, a strong positive correlation was shown by serum ceruloplasmin levels in group I and group II ( $r=+0.9)$. Ironically the values in group III of ceruloplasmin were positive the correlation was not strong. The Malondialdehyde values in group III also showed a strong positive correlation with $r$ values of +0.93 . In the hsCRP group, a strong positive correlation was found in group III however, a weak positive correlation was found in group I and group II.

Materials and Methods: A Hospital-based Prospective study was conducted in the Department of Medicine, Santhiram medical college \& general hospital for a 2 year period.Universal Sampling Technique was used for the selection of study subjects.The study population included patients admitted with fever, flank pain, and positive urine or blood cultures in the department of general medicine in Santhiram medical college and general hospital.The final sample size was 50 subjects.

Conclusion: Serum ceruloplasmin was positively correlated with PASI scores and serum MDA levels were highly correlated in severe cases of psoriasis. The hs-CRP was also found to be elevated in the psoriasis cases as compared to controls however, a significant positive correlation was found in severe cases. Therefore, psoriasis severity can be monitored by estimating the biomarkers, especially in severe cases.
Keywords: Serum ceruloplasmin, Malondialdehyde (MDA), hsCRP, Psoriasis Area and Severity Index (PASI)

\section{INTRODUCTION}

Psoriasis is a chronic, recurrent, non-infectious disease of the skin with erythematous plaquesdistributed over extensor surfaces of knees, elbows, buttocks, palms, and scalp. The pathophysiology of psoriasis is still not very clear. The main abnormality in psoriasis appears to be increased epidermal proliferation due to excessive division of cells in the Basal layer. Epidermalhyperproliferation accounts for many of the metabolic abnormalities including alteration inthe serum levels of proteins and some trace elements. Psoriasis is estimated to affect $2-4 \%$ of the population of the western world. The rate of psoriasis varies according to age, region, and ethnicity; a combination of environmental and genetic factors is thought to be responsible for these differences. ${ }^{[1]}$ Although not completely understood, there is a genetic component in the development of psoriasis. Twin studiesshow a $67 \%$ concordance for monozygotic twins versus $18 \%$ for dizygotic twins. This concordance in monozygotic twins suggests multifactorial inheritance andinteraction between genetic predisposition and the environment factors are involved.Psoriasis affectsboth sexes equally. ${ }^{[2]}$ Psoriasis is more common in countries farther from the equator. ${ }^{[3]} \mathrm{However}$, there issometimes anobvious environmental trigger such as infection. Oxidative stress is linked with the severity of psoriasis Malondialdehyde (MDA) is the marker of oxidative stress. ${ }^{[4]}$ Ceruloplasmin is an acute phase reactant that has a role in scavenging oxygen-derived free radicals. ${ }^{[5]}$

Purified human ceruloplasmin has been shown to inhibit the oxidation of tissue extracts of lipids, polyunsaturated fatty acids, and phospholipids. Ceruloplasmin's antioxidant activity also blocks protein and DNA damage and removes reactive intermediates that, in the case of hydrogen peroxide, a membrane-permeable oxidant, could lead to an altered intracellular redox state of vascular cells. ${ }^{[6,7]}$ CRP is an acutephase reactant protein derived from hepatocytes and it is synthesis is controlled to a large extent by IL-6, but IL-1 and TNF-aits activity is increased in pro-inflammatory states in the body. ${ }^{[8]}$ Increased levels of biochemical markers suggest a relationship between psoriasis, oxidative damage, and 
inflammation. Inflammatory markers particularly malonaldehyde (MDA),hs-CRP, and Ceruloplasmin can be used to assist inassessing disease severity and response to treatment in a patient with psoriasis. With this background, we in the current study tried to determine the role of malondialdehyde (MDA), ceruloplasmin, and C-Reactive Protein (hs-CRP) as markers of severity of psoriasis.

\section{Material and Methods}

This cross-sectional study was undertaken at RIMS Adilabad. Institutional ethical committee permission was obtained after duly following protocol for human studies. Written consent was obtained from all the participants of the study after explaining the nature of the study in their local language.

\section{Inclusion criteria}

1. Successive Patients diagnosedwith psoriasis

2. Aged between $20-45$ years

3. Those willing to participate in the study and available for follow-up

\section{Exclusion criteria}

1. Those on medication for psoriasis/PUVA therapy

2. Patients with bacterial/viral infections

3. Hypertension

4. Malignancy

5. Smoking

6. Other autoimmune skin diseases (SLE)

After selection of the case to be included in the study a detailed history which included past- history, family history of similar disease, personal history, and drug intake was obtained as per the proforma. Each patient was graded for Psoriasis Area and Severity Index (PASI) score and were segregated into various groups. Group, I was with a PASI score of $<10$ where mild cases $\mathrm{n}=25$ cases were included. Group II was with PASI Scores of 1115 were moderate cases $(n=25)$. Group III with PASI >15 were severe cases $(n=25) . N=25$ age and sex-matched taken from healthy people were included in group IV controls. Blood samples were collected between 10:00 am to 11:00 am. Venous blood $5 \mathrm{ml}$ obtained in a sterile needle and syringe from the antecubital vein under aseptic precautions. The blood samples were then transferred to clean sterile centrifuge tubes and allowed to clot. The clotted samples were centrifuged at 3000 rpm for 5 minutes to obtain serum. The serum was taken using micropipette and Eppendorf tubes. The biochemical assays were carried out within $24 \mathrm{hrs}$ of collection of samples. Ceruloplasmin was estimated by the Houchin method [9] using Para Phenyl Diamine acetate buffer and sodium azide. MDA was measured by the Mahalouz method [10] using 20\% TCA and $0.67 \%$ TBA. CRP was estimated by turbidimetric immunoassay [11] in a semi-automated analyzer. All the data obtained was entered in MS Excel and analyzed using SPSS version 19 on windows format.

\section{Results}

Out of $n=75$ cases of study groups, $n=40$ were females and $n=35$ males. In the group-I $n=12$ male and $n=13$ females. In group II $n=10$ males and $n=15$ females and group III $n=13$ males and $n=12$ females were enrolled. In group IV the control group of $n=11$ male and $n=14$ females were included. The mean age group of all the males in the study groups was $31.5 \pm 1.5$ years the mean age of females was $27.5 \pm 1.0$ years. In the control group, the mean age of males was $30.0 \pm 1.0$ and females was $26.5 \pm 1.0$ years.

The mean levels of serum ceruloplasmin were highest in study group III which had the highest mean PASI scores of $16.16 \pm$ 1.28 and lower values in group I with mean PASI scores of 6.04 \pm 1.83 . The values of ceruloplasmin in group II with mean PASI scores of $12.4 \pm 1.32$ were intermediate between group I and group III. The least values of ceruloplasmin were found in the control group IV. The MDA values also showed an increasing trend from the group I to group III and least values in group IV control group. The mean hs-CRP values were $>2$ in group II, hs-CRP >3 in group II and group III, and the values of control group IV were 0.83 given in table 1.

Table 1: The mean value of parameters recorded in different groups of patients

\begin{tabular}{|c|c|c|c|c|c|}
\hline Groups & $\begin{array}{c}\text { No of } \\
\text { cases (N) }\end{array}$ & $\begin{array}{c}\text { Ceruloplasmin } \\
(\mathrm{mg} / \mathrm{dl})\end{array}$ & $\begin{array}{c}\text { MDA } \\
(\mathrm{nmol} / \mathrm{ml})\end{array}$ & $\begin{array}{c}\text { hs-CRP } \\
\text { (mg/L) }\end{array}$ \\
\hline \multirow{2}{*}{ Group I } & 25 & $\begin{array}{c}\text { Mean } \pm \\
\text { SD }\end{array}$ & $\begin{array}{c}39.38 \\
8.34\end{array}$ & $\begin{array}{l}6.49 \\
1.28\end{array}$ & $\begin{array}{c}2.36 \\
0.28\end{array}$ \\
\hline \multirow{2}{*}{ Group II } & \multirow{2}{*}{25} & Mean \pm & 53.31 & 6.78 & 3.06 \\
& & SD & 5.29 & 0.87 & 0.42 \\
\hline \multirow{2}{*}{ Group III } & \multirow{2}{*}{25} & Mean \pm & 67.08 & 7.14 & 3.50 \\
& & SD & 1.916 & 1.52 & 0.37 \\
\hline \multirow{2}{*}{ Group IV } & \multirow{2}{*}{25} & Mean \pm & 23.88 & 2.51 & 0.83 \\
& & SD & 1.83 & 0.29 & 0.13 \\
\hline
\end{tabular}

A graph plotted of mean serum values of ceruloplasmin versus PASI in all groups showed an increasing trend of ceruloplasmin values with increasing scores of PASI as depicted in figure 1. The least values of the left hand recorded were in group IV and the highest values of the right-hand side in group III.

Figure 1: Showing the mean serum ceruloplasmin versus PASI in all groups

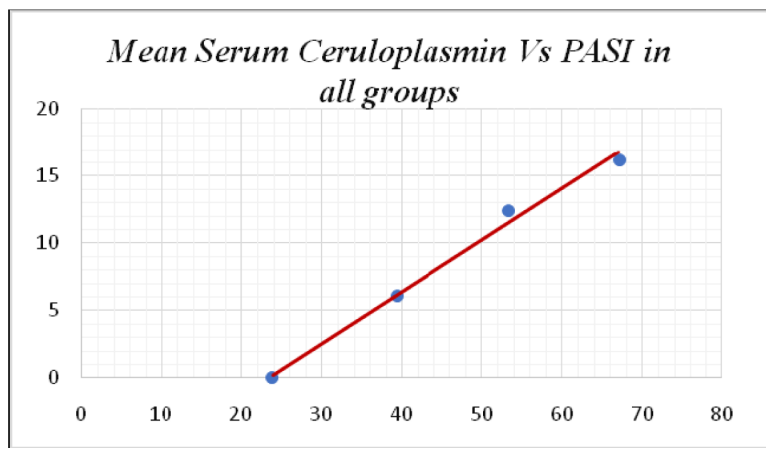


The mean Malondialdehyde values were plotted with PASI in all groups with the least values of MDA in group IV and highest in group III. Although, among the study groups the variation appeared to lesser however values were far greater than the control group given in figure 2.Figure 3 shows the mean values of hs-CRP recorded in all the groups. The values in group I, group II and group III were ranging from 2.3 to 3.5 and the values in group IV were < 1 (0.8).

The Pearson correlation coefficient $(r)$ values were calculated between PASI and biomarkers. Of the biomarkers, a strong positive correlation was shown by serum ceruloplasmin levels in group I and group II ( $r=+0.9)$. Ironically the values in group III of ceruloplasmin were positive the correlation was not strong. The Malondialdehyde values in group III also showed a strong positive correlation with $r$ values of +0.93 . In the hsCRP group, a strong positive correlation was found in group III however, a weak positive correlation was found in group I and group II.

Figure 2: Showing the mean serum MDA versus PASI in all groups

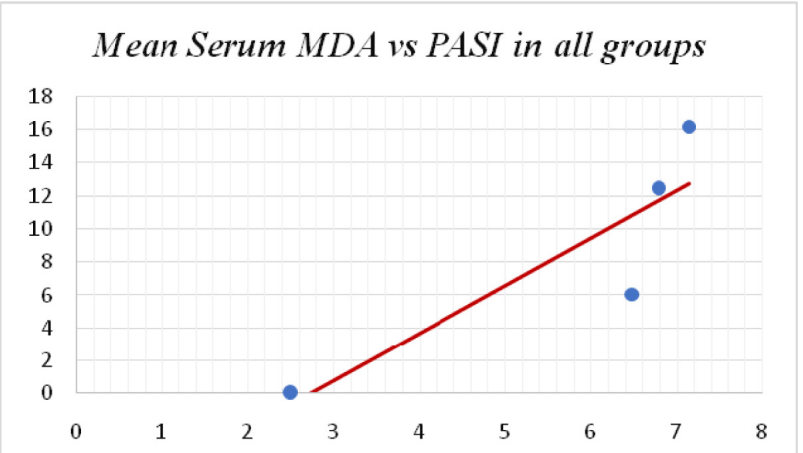

Figure 3: Showing the mean serum hs-CRP versus PASI in all groups

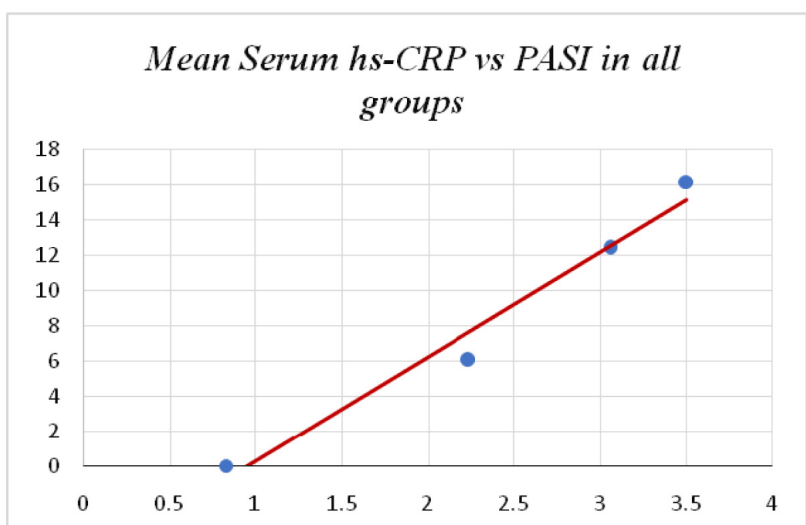

Table 2: Correlation coefficient $(r)$ values of various parameters with PASI scores

\begin{tabular}{|c|c|c|c|}
\hline \multirow{2}{*}{ Category } & \multicolumn{3}{|c|}{ PASI versus } \\
\cline { 2 - 4 } & Ceruloplasmin & MDA & hs-CRP \\
\hline Group I & 0.90 & 0.756 & 0.185 \\
\hline Group II & 0.90 & 0.662 & 0.215 \\
\hline Group III & 0.418 & 0.932 & 0.728 \\
\hline
\end{tabular}

Note: all the correlation values are Positive (+)

\section{Discussion}

Psoriasis is a common inflammatory and proliferative disease of the skin. Thecurrent gold standard for the assessment of extensive psoriasis has been PsoriasisArea and Severity Index (PASI) scores. However,biomarkers can be very useful in monitoring the response to treatment and prognosis of the disease. In this study, we observed a positive correlation between serum ceruloplasmin, MDA, and hs-CRP with PASI scores. MS Dadras et al; ${ }^{[12]}$ investigating Serum Copper, Iron, Ceruloplasmin, and Transferrin Levels in Mild and Severe Psoriasis Vulgaris in Iranian Patients found the serum Fe, Trf, and $\mathrm{Cu} / \mathrm{Cp}$ levels of the patients with psoriasis were statistically lower compared with those of the controls; serum levels of ceruloplasmin was elevated in patients with psoriasis compared to controls. In the current study we found elevated levels of ceruloplasmin in psoriasis cases as compared to controls. An increase in ceruloplasmin [Cp] levelsmight be due to increased inflammation in the active phase of the disease. The increased or decreased levels of measured trace elements and proteins may participate in the pathogenesis of psoriasis via ameliorating inflammation, producing ROS, or removing inhibition on the immune system. ${ }^{[13]} \mathrm{C}$ Shenoy et al;[5]investigating serum ceruloplasmin levels with psoriasis severity found the mean ceruloplasmin levels of cases were found to be higher comparedto controls and it was statistically significant. But they found a mild negative correlation between ceruloplasmin and severity index PASI scores in contrast to the results of this study.Serum malondialdehyde (MDA) is one of the products of polyunsaturated fatty acid peroxidation. It can impair the physiological mechanisms of the body because of its ability to interact with DNA and proteins.The reactive oxygen species derived from neutrophils, keratinocytes, and fibroblasts contribute to neutrophil action and play role in the psoriatic process. ROS acts as second messengers in the induction of biological responses by activation of NF-kB or AP-1, generation of cytokines, modulation of signaling pathways, and activation of peroxisomesproliferator-activated receptors. ${ }^{[14,15]} \mathrm{We}$ in the current study found the highest positive correlation of MDA with group III indicating severe psoriasis increases MDA levels it is in concordance with other studies in this field they have observed similar findings. ${ }^{[11,15,16]}$ Serum hs-CRP which is a marker of systemic inflammation was found to higher in psoriasis cases as compared to controls in this study a higher 
correlation of hs-CRP with group III cases was found. AR Vadakayil et al; ${ }^{[17]}$ in their study found significantly higher CRP levels in cases of psoriasis as compared to controls. They concluded that CRP may be considered as a useful marker of psoriasis severity that could be used to monitor psoriasis and its treatment. PR Pereira et al; ${ }^{[18]}$ found elevated levels of CRP in severe psoriasis cases and concluded that neutrophils play a crucial role by contributing to the development of oxidative and proteolytic stress. The worsening of the disease seemed to be linked to the enhancement of the inflammatory response and the imbalance between neutrophil activation products and their inhibitors.

\section{Conclusion}

Within the constraints of the present study, it can be concluded that there is a positive association between the biomarkers and the severity of psoriasis. Serum ceruloplasmin was positively correlated with PASI scores and serum MDA levels were highly correlated in severe cases of psoriasis. The hs-CRP was also found to be elevated in the psoriasis cases as compared to controls however, a significant positive correlation was found in severe cases. Therefore, psoriasis severity can be monitored by estimating the biomarkers, especially in severe cases.

\section{REFERENCES}

1. Parisi R, Symmons DP, Griffiths CE, Ashcroft DM (February 2013). Identification and Management of Psoriasis and Associated Comorbidity (IMPACT) project team. Global epidemiology of psoriasis: a systematic review of incidence and prevalence. J Invest Dermatol 2013;133(2): 377-85.

2. Kruger G. Ellis CN. Psoriasis- recent advances in understanding its pathogenesis and treatment.J Am Acad Dermatol 2005; 53(Suppl 1): S94-100.

3. Guerra I, Gisbert JP. Onset of psoriasis in patients withinflammatory bowel disease treated with anti-TNF agents. Expert RevGastroenterol Hepatol2013;7(1): 4148.

4. Rocha-Pereira P, Santos-Silva A, Rebelo I, Figueiredo A, Quintanilha $F$, Teixeira $A$. The inflammatory response in mild and in severe psoriasis. BJD. 2004; 150(5): 917-928.

5. Chetana Shenoy, ManjulaShantaram, Kalashree K, Neevan D.R. D'Souza, Manjunath Shenoy M. A study of ceruloplasmin in psoriasis and its correlation with disease severity. International Journal of Analytical, Pharmaceutical, and Biomedical Sciences. 2013;2(4):3335.

6. Danxia Liu, Jing Wen, Jing Liu, Liping L. The roles of free radicals in amyotrophic lateral sclerosis: Reactive oxygen species and elevated oxidation of protein, DNA, and membrane phospholipids. The FASEB Journal 2000; 13(15):2318-28.
7. Mukhopadhyay CK. Mazumder B. Lindley P. Fox PL. ProcNatl Acad Sci USA 1997; 94:11546-51.

8. Flatz L, Conrad C. Role of T-cell-mediated inflammation in psoriasis: pathogenesis and targeted therapy. Psoriasis: Targets and Therapy 2013; 3:1-10.

9. Giurgea N, Constantinescu MI, Stanciu R, Suciu S, Muresan A. Ceruloplasmin - acute-phase reactant or endogenous antioxidant? The case of cardiovascular disease. Med Sci Monit. 2005;11:48-51.

10. Rocha-Pereira P, Santos-Silva A, Rebelo I, Figueiredo A, Quintanilha $F$, Teixeira $A$. The inflammatory response in mild and in severe psoriasis. BJD. 2004; 150(5): 917-928.

11. Gutteridge JM, Stocks J. Ceruloplasmin: physiological and pathological perspectives. Crit Rev Clin Lab Sci 1981; 14: 257-329.

12. MS Dadras, N Namazi, S Younespour. Comparative Analysis of Serum Copper, Iron, Ceruloplasmin, and Transferrin Levels in Mild and Severe Psoriasis Vulgaris in Iranian Patients. Indian Dermatol Online J 2017; 8(4): 250-253.

13. M Mittal, MR Siddiqui, K Tran, SP Reddy, AB Malik. Reactive Oxygen Species in Inflammation and Tissue Injury. Antioxidants and Redox Signaling 2014;20(7): 1126-30.

14. Rashmi R, Rao KS, Basavaraj KH. A comprehensive review of biomarkers in psoriasis. Clin ExpDermatol. 2009; 34(6):658-663.

15. Manjula S, Aroor AR, Raja A, Rao SN, Rao A. Elevation of serum ceruloplasmin levels in brain tumors. 1992; 86(2):156-158.

16. Bacchetti T, Campanati A, Ferretti G, Simonetti O, Liberati G, Offidani AM. Oxidative stress and psoriasis: the effect of antitumor necrosis factor-a inhibitor treatment. $\mathrm{Br} \mathrm{J}$ Dermatol. 2013 May; 168(5):984-989.

17. AR Vadakayil. S Dandekeri, SM Kambli, NM Ali. Role of Creactive protein as a marker of disease severity and cardiovascular risk in patients with psoriasis. Indian Dermatol Online J 2015; 6(5):322-25.

18. PR Pereira, AS Silva, I REbelo, A Figueriedo, A Quintanilha, $F$ Teixeria. The inflammatory response is mild and in severe psoriasis. British Journal of Dermatology 2004;150(5): 917-28.

How to cite this article : K Prashanth, Khan MI. Study of serum Ceruloplasmin, Malondialdehyde (MDA) and hs-CRP as biomarkers of severity in Psoriasis. Perspectives in Medical Research 2020; 8 (3):70-73.

DOI: $10.47799 /$ pimr.0803.15

Sources of Support: Nil, Conflict of interest: None declared 